\title{
Chitosan cushioned air stable single PEGylated phospholipid bilayers
}

\author{
Y. B. Zhang ${ }^{1,2}$, Y. Y. Chen ${ }^{1,3}$, Z.J. Ding ${ }^{1}$, C. X. Wang 1 , C. H. Huang1,2 and G. Jin 4 \\ ${ }^{1}$ Suzhou Institute of Nano-tech and Nano-bionics, CAS, Jiangsu, 215125, P. R. China; \\ ${ }^{2}$ Graduate University of CAS, Beijing, 100049, P. R. China; \\ ${ }^{3}$ Institute of Biophysics, CAS, Beijing, 100101, P. R. China; \\ ${ }^{4}$ Institute of Mechanics, CAS, Beijing, 100190, P. R. China
}

\begin{abstract}
Air stable single lipid bilayers formation through SUVs fusion methods is still a challenge work. Here soluble LMW chitosan chemically coupled to silicon dioxide surface is cushioned for PEGylated lipid bilayers. Using imaging ellipsometry and spectroscopic ellipsometer, the influence of molar fraction of DSPE-PEG ${ }_{2000}$ for lipid bilayers formation and stability is examined. Under the mushroom phase, PEGylated lipid vesicles may aggregate to multiple bilayers; while entering the brush regime of PEG, vesicles can fused into single lipid bilayers. It is demonstrated both the appropriate mole fraction of PEGylaed lipid and hydrophilic polymer cushion are equally critical for achieving air and wash stable single lipid bilayers. Imaging ellipsometry also show the promise as an easy and useful tool for future air stable lipid bilayers based biosensor.
\end{abstract}

Keywords - air stable, single phospholipid bilayers, chitosan, imaging ellipsometry

\section{InTRODUCTION}

Artificial biological membranes-supported phospholipid bilayers (SPB) have been used as model membranes, e.g., to study cell-cell recognition in the immune system [1], adhesion of cells [2] and membrane proteins or ion channels based biosensors [3]. Small unilamellar vesicle (SUVs) is used widely for SPB preparation since its easier manipulation and handling. However, vesicles would form multiple lipid bilayers rather than single bilayers after adsorption and fusion onto substrate, which might be troublesome sometimes in the application. Most of published research works on phospholipid membrane are mainly using fluorescence labeling and fluorescence recovery after photobleaching (FRAP) technology, which is never telling the detailed structure of lipid bilayers. In an effort to overcome this problem, we recently designed a polymer cushioned PEGylated lipid membrane for future application in protein sensor. With the hand of imaging ellipsometry, we can examine the thickness of formed lipid bilayers and discriminate the single bilayers or not.

\section{Materials AND METHODS}

Materials

1,2-Distearoyl-sn-Glycero-3-Phosphoethanolamine-N[Methoxy(Polyethylene glycol)-2000] (DSPE-PEG 2000 ), 1,2Dimyristoylphophatidylcholine (DMPC) were products of AvantiPolar Lipid, Inc., Alabaster, AL. 3aminopropyltriethoxysilane (APTES) were purchased from Acros Organics, Belgium. LMW chitosan (MW=10, 000, degree of deacetylation: $85.31 \%$ ) was a gift from Jinan Haidebei Marine Bioengineering Co., China.

\section{Methods}

\section{LMW chitosan modified silicon substrate}

Polished silicon wafers was cleaned with pirahna solution and further modified with APTES according to the protocols described before [4]. NHS activating succinic acids was used as cross-linkers for surface coupling of LMW chitosan.

PEGylated lipid membrane formation

SPB was formed through extruded SUVs fusion method [5]. The substrate dotted with SPB was washed with deionized water and dried with pure nitrogen gas before further detection with imaging ellipsometry or thickness measurement with Woollam M-2000 spectroscopic ellipsometer.

\section{Imaging ellipsometry}

The phospholipid membrane array was detected by imaging ellipsometry which was detailed in other places [6]. It was used a CCD camera to image the ellipsometry response of a larger area sample with lateral distribution in different layer thickness, and the result was grabbed as a digital image and stored in a computer with a grayscaleformat ( 8 bits, 0-255 grayscale) for further evaluation by an image-processing program.

\section{RESULTS AND DISCUSSION}

Air-stable chitosan cushioned PEGylated lipid membrane 
Piranha solution treatment can make silicon surface super hydrophilic, with a contact angle lower than $5^{\circ}$. After modified with APTES, the surfaces will loss some extent of hydrophilicity and the contact angle was about $55^{\circ}$. It was reported that either PEG [7] or $\gamma$-aminopropylsilane [8] could enhance the air stable of lipid membrane. However, neither PEG nor APTES used in our experiments succeed in showing the air stability of lipid membrane. After doting vesicles on the three different supports: super hydrophilic silicon surface, APTES modified silicon and LMW chitosan coupled surface, only chitosan cushioned PEGylated membranes gave visible lipid layers after washing and drying procedure (Fig. 1).

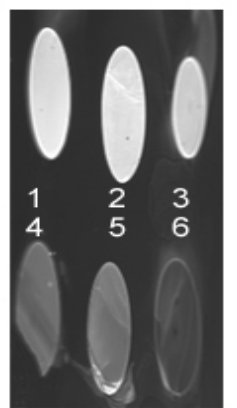

Fig. 1 Air-stable chitosan cushioned PEGylated lipid membrane. The numbers indicate several molar contents of DSPE-PEG ${ }_{2000}$, which are 0.1, $0.25,0.5,1.5,2.5,5 \mathrm{~mol} \%$ successively.

Here we proposed a possible reason for such interesting results as potentially unsymmetrical distribution of PEG in the upper and down layer of lipid membrane. It is not difficult to imagine the steric hindrance of PEG molecules within vesicles, so more PEG moieties may prefer to occupy the outlayers of vesicles. Both Salafsky [9] and Contino [10] had shown that the orientation of the leaflets was preserved upon fusion of proteoliposomes to plain glass. In that case, the outlayer of vesicle should become upper layer of lipid membrane after vesicles adsorption on support, which results in the uneven amount of PEG between upper and lower bilayers. If it is true for above hypothesis and the PEG is in mushroom phase, the much less PEG of lower layer will inevitably fail protecting the lipid membranes when they are exposed to the air/water interface. While the hydrophilic chitosan cushion could increase the bending elastic modulus of the lipid membrane and thus increase the barrier for lipid patches to roll up into sheets and peel away as vesicles.

\section{Single PEGylated phospholipid bilayers}

As shown in the Fig. 2, the grayscales of PEGylated lipid membrane decreased while the molar fraction of PEG was increased. SUVs containing PEG lower than $1.5 \mathrm{~mol} \%$ gave much brighter membranes. The grayscale of lipid membranes containing $0.1 \mathrm{~mol} \%$ reached as high as the ultimate value. From our observation, we concluded that the membrane was comprised of bilayer aggregates and/or unfused vesicles.

We got a good linear region after curving the thickness of lipid membranes against grayscale values. After calculating thickness of lipid membrane with series PEG content, it was found the membrane thickness was gradually reduced to the normal single bilayers as increasing PEG molar fraction. A supported lipid bilayer is characterized by having a thickness of 4-5 nm. [11] However, the dried PEGylated lipid membrane also showed a thickness around $4 \mathrm{~nm}$ (Fig. 3). The polymer moieties have little impact on the thickness of lipid membrane when they are all in dehydration environment. It maybe due to hydrophilic polymer cushion and PEG shielding both upper and down layer, lipid membrane could keep stable in the air condition. (Fig. 4)

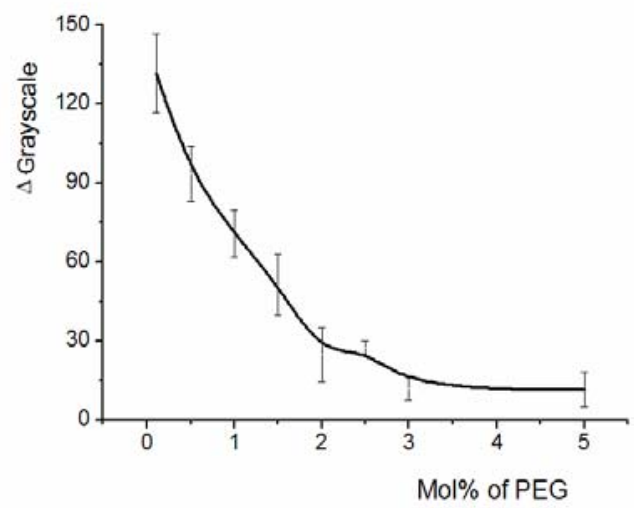

Fig. 2 Grayscales values addition against mole fraction of DSPE-

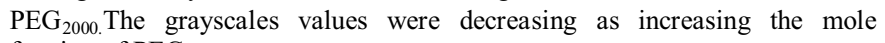
fraction of PEG.

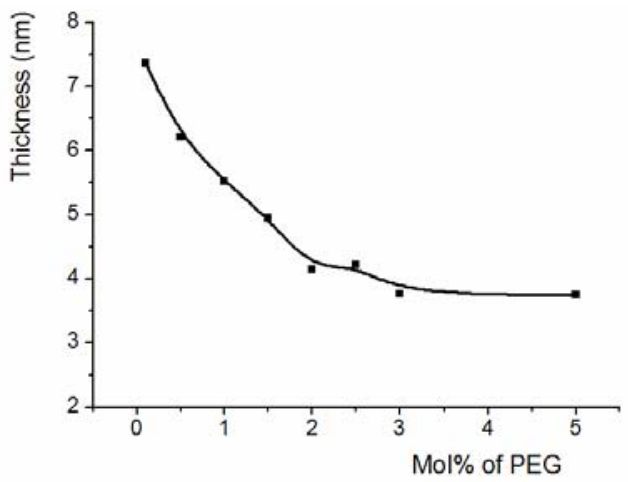

Fig. 3 Membrane thickness against mole fraction of DSPE-PEG ${ }_{2000}$. The lipid membrane thicknesses were reducing while raising PEG content. 


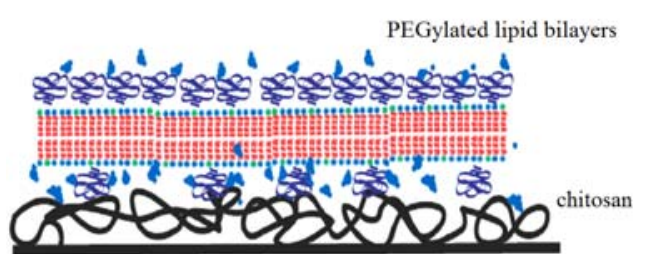

Fig. 4 Schematic chitosan cushioned lipid bilayers.

The transition from mushroom to brush phase happened when molar fraction of $\mathrm{PEG}_{2000}$ reached $1.4 \mathrm{~mol} \%$ [12]. Within the mushroom phase, PEG was in too low density and had little power of stealth effect [13], which was an ability of a PEG-grafted layers to prevent close approach to the liposome surface. In the absence of sufficient grafted polymer, vesicles will adhere to existing lipid bilayers to form multilayer through van der Waals' attraction [14]. As a result, the thickness or grayscales of lipid membrane was much higher than that of single bilayers. When the hydration repulsion of PEG molecules between vesicles and bilayers were improved as raising the PEG amount, the vesicles in the solution would cease to adsorb or fuse onto the formed lipid membrane and the single bilayers could be maintained.

But it seemed too early to say the single lipid bilayer could be formed only if the PEG molar fraction was added above its mushroom-to-brush phase transition concentration. We found PEGylated lipid membrane would be washed away if PEG was added more than $5 \mathrm{~mol} \%$, since there was no obvious grayscales addition or thickness detection. We thought that was caused the interactions between polymers and water molecules. Under the vigorously or repeated washing force, PEG will be pulled out from lipid structure, inducing the latter deformation and depletion of lipid membrane.

\section{Conclusions}

Here a simple and facile method is used for air and wash stable single lipid bilayer formation. Results demonstrate that the surface density of DSPE-PEG 2000 is a critical parameter in single lipid bilayers formation visualized by using imaging ellipsometry and spectroscopic ellipsometer. It is suggested the proper molar fraction of PEG for getting single lipid bilayers is around at its mushroom-to-brush phase transition. Furthermore, taking hydrophilic polymers as lipid cushion, like chitosan, is also a key for keeping air stability of lipid membrane.

\section{ACKNOWLEDGMENT}

We thank Mr. Tian of Jinan Haidebei Marine Bioengineering Co. for kind gift. The authors would like to gratefully acknowledge financial supports from National Basic Research
Program of China 2009CB320300, National High Technology Research and Development Program (863) of China, National Natural Science Foundation of China( 10804083, 20845003), Chinese Academy of Sciences((KJCX2.YW.M02 and M04)) and National Post-doctoral Funds.

\section{REFERENCES}

1. McConnell H M, Watts T H, Weis R M et al. (1986) Supported planar membranes in studies of cell-cell recognition in the immune system. Biochim Biophys Acta 864:95-106.

2. Stelzle M and Sackmann E. (1989) Sensitive detection of protein adsorption to supported lipid bilayers by frequency-dependent capacitance measurements and microelectrophoresis. Biochim Biophys Acta 981:135-142. 3. Castellana E T and Cremer P S. (2006). "Solid supported lipid bilayers: From biophysical studies to sensor design." Surf Sci Rep 61: 429-444.

4. Wang Z H and Jin G. (2004) Covalent immobilization of proteins for the biosensor based on imaging ellipsometry. J Immunol Methods 285:237- 243. 5. Lapinski M M, Forero A C, Greiner A J et al. (2007) Comparison of liposomes formed by sonication and extrusion: rotational and translational diffusion of an embedded chromophore. Langmuir 23:11677-11683.

6. Jin G. (2008). Development of biosensor based on imaging ellipsometry. Phys Stat Sol (a) 205:810-816.

7. Albertorio F, Diaz A J, Yang T et al. (2005) Fluid and Air-Stable Lipopolymer Membranes for Biosensor Applications. Langmuir 211:74767482 .

8. Fang Y. (2006) Spreading and segregation of lipids in air-stable lipid microarrays. J Am Chem Soc 128:3158 -3159.

9.. Salafsky J, Groves J T and Boxer S G. (1996) Architecture and function of membrane proteins in planar supported bilayers: a study with photosynthetic reaction centers. Biochemistry 35:14773-14781.

10. Contino P B, Hasselbacher C A, Ross J B et al. (1994) Use of an oriented transmembrane protein to probe the assembly of a supported phospholipid bilayer. Biophys J 67:1113-1116.

11. Castellana E T and Cremer P S. (2006) Solid supported lipid bilayers: From biophysical studies to sensor design. Surf Sci Rep 61:429-444.

12. Needham D, Kim D H. (2000) PEG-covered lipid surfaces: bilayers and monolayers. Colloid Surfacce B 18:183-195.

13. Lasic D D and Needham D. (1995) The" Stealth" Liposome: A prototypical biomaterial. Chemical Reviews 95:2601-2628.

14. Cevc G and Marsh D. (1987) Phospholipid Bilayers. Physical Principles and Models. Wiley-Interscience, New York.

Corresponding Author: Gang Jin

Institute: Institute of Mechanics, Chinese Academy of Sciences

Street: \#15, Bei-si-huan west Road

City: Beijing 100190

Country: P.R. China

Email: gajin@imech.ac.cn 